\title{
Multi-Player Virtual Ping-Pong Game
}

\author{
Young-Bum Kim, Seung-Hoon Han, Sun-Jeong Kim, Eun-Ju Kim, Chang-Geun Song \\ Div. of Information Engineering and Telecommunications \\ Hallylm University \\ 39 Hallymdaehak-gil, Chuncheon, Gangwon-do, 200-702 Korea \\ \{stylemove, issess, sunkim, ejkim628, cgsong\}@ hallym.ac.kr
}

\begin{abstract}
In this paper we show how a motion capture system and feedback mechanism can be integrated into a virtual pingpong game to create a multi-player platform. To trace the motion of each player, optical markers are attached to different places on each player's paddle. For tactile feedback, we designed a controller for a DC (Direct Current) motor, which is also attached to the paddle. This controller communicates with the game server through wireless Bluetooth technology. When the game server detects a collision between the paddle and ball, the controller receives the message from the game server and then triggers one of the respective paddle's DC motors to vibrate depending on the position of the impact on the paddle. During an exhibition many people positively responded to the game.
\end{abstract}

\section{Introduction}

While computers and other interaction technologies provide exciting directions for game design and play, computer games tend to eliminate many of the social aspects of game play. Whereas playing non-electronic games (e.g. board games) is mostly a collective activity involving more than one person in direct interaction, playing computer games is often an individual activity [6]. Our motion capture system opens up a range of new application areas for computer games. One such class of applications is Squidball that is a real-time interactive game using motion capture technology [1]. It traces the balloons' spatial location to facilitate interaction between players and balloons for a cooperative and energetic game.

A virtual tennis game by VR devices utilized headmounted displays, magnetic sensors, and data gloves to bring interactivity to the virtual tennis environment [4]. Their motion capture equipment interfered with game play as it restricted player motion. Another virtual ping-pong game called V-Pong solved this problem by utilizing an op- tical motion capture system [2], but this game lacked multiplayer capability. We have explored how optical sensing technology can be utilized in the design of multi-player games. We attached optical markers to different parts of each player's paddle so that we could obtain motion data of each player separately. To help capture the motion of players, optical markers were also attached to wireless headsets. Visual, audio, and tactile feedback systems were employed to improve the playing experience. For tactile feedback, we attached a digital wireless controller to the paddle which triggers DC motors to vibrate the paddle simulating the feeling of actual contact with a ball. Let us summarize the main features:

- The optical sensing system for multi-player ping-pong game

- The tactile feedback system using DC motors as well as visual and audio feedback from the computer

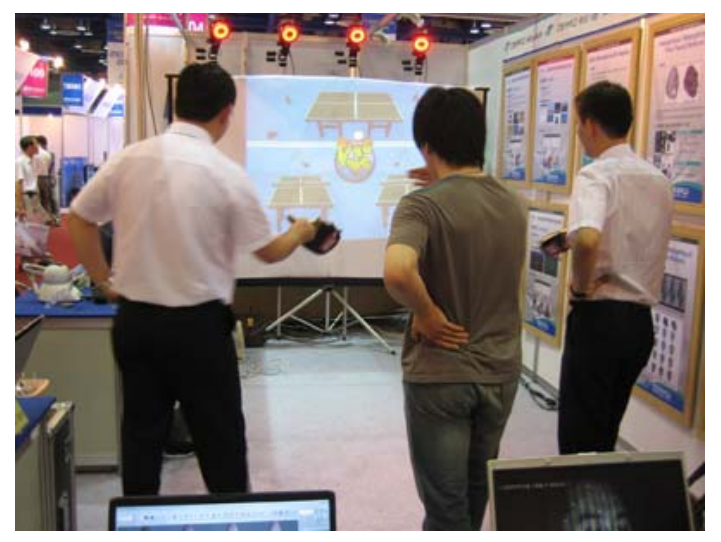

Figure 1. Players enjoyed our virtual pingpong game during a demonstration at SEK2007 Exhibition, COEX Seoul, Korea. 


\section{System Overview}

Our multi-player virtual ping-pong game consists of the optical motion capture equipment, a game server, the devices for display, two paddles, and two wireless headsets (Figure 2). The optical motion capture equipment has four infrared cameras and two PCs. The cameras sense optical markers attached to the paddles and headsets of players. One PC collects the image data from these cameras while the other PC analyzes the image data and produces the motion data. The game server receives the binary format motion data from the motion capture equipment through TCP/IP protocol and computes visual, audio, and tactile feedback. Visual feedback is the display the movement of paddles and a ball on a video screen. Audio feedback is the sound players hear when the ball is struck. Tactile feedback is the vibration a player feels in the paddle when they hit the ball.

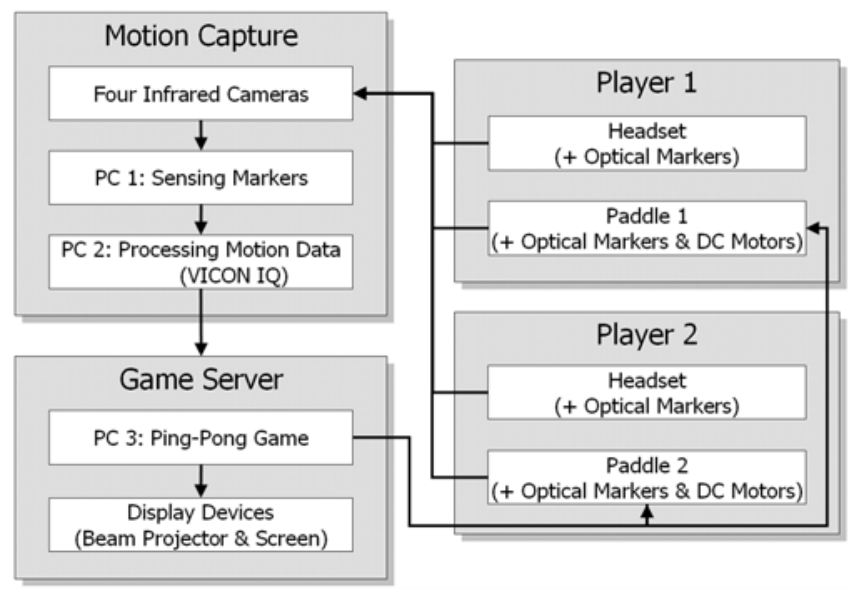

Figure 2. System overview

\section{Motion Capture for Multi-Player Game Play}

Here we describe the challenges of building motion capture tools the multi-player virtual ping-pong game.

\subsection{Motion Capture Setup}

We utilized the VICON motion capture system [5] (Figure 3). The system can track motion capture markers within a capture distance of approximately $5 \sim 6$ meters. The motion markers are made of retro-reflective material. Visible illuminators placed around the camera lens emit light which the visual markers reflect back into the camera. This makes the retro-reflective markers appear significantly brighter to the camera than other objects. Image processing (thresholding and circle fitting) is used to track those markers in each view. Triangulation of multiple camera views results in very accurate and robust 3D marker tracking.
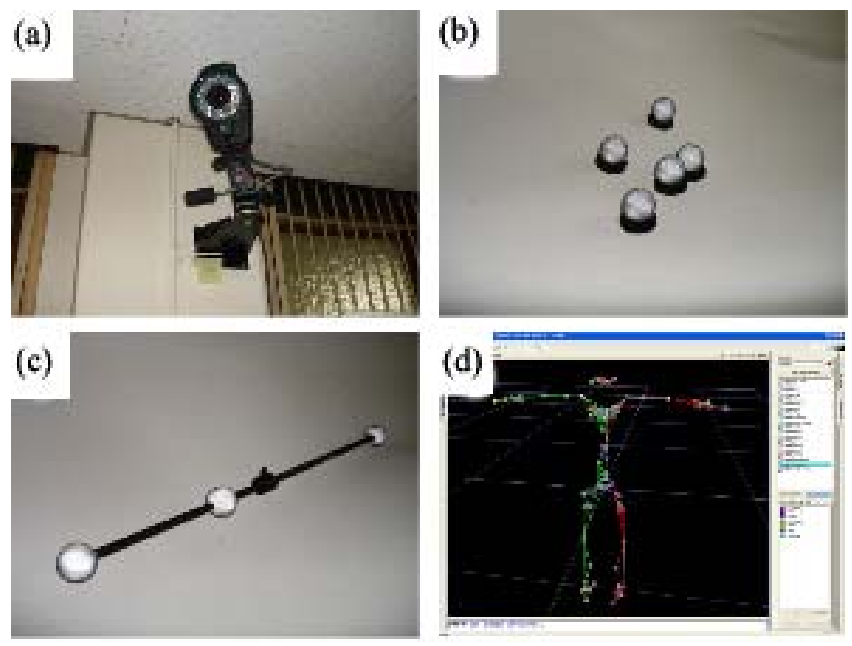

Figure 3. VICON motion capture system: (a) Infrared camera, (b) Retro-reflective markers, (c) Calibration wand, (d) Screen shot of VICON IQ

To set up the motion capture system, cameras must be set up and calibrated. Camera placement for a motion capture system is an iterative refinement process dependent on several site-specific aspects. For motion capture, cameras are usually placed in rectangular perimeter on the ceiling around the capture space. We used this method in our laboratory but in the exhibition four cameras were placed on the corners of the front wall facing players. Both placements were functional. The final step in setting up the system is camera calibration. Using the VICON software the calibration process is done by waving a calibration object throughout the entire capture area. This is usually a wand that has two or three retro-reflective markers placed in a straight line. The 2D-tracking data for the calibration object from each camera is then used to compute the exact 3D locations.

\subsection{Ping-Pong Paddles}

The motion capture equipment requires spherical markers for effective tracking. Figure 4 shows that the optical markers are attached to two paddles in different way. The difference of the marker positions enables the motion capture equipment to track paddles when they are overlapped in the camera view. A set of the markers needs a VST (VICON 
Skeleton Template) file to abstract the structure of the markers. If the default VST file is used, the position and rotation angle of the markers are not accurate. Therefore we built a new VST file for use with two paddles in a multi-player virtual ping-pong game situation. To obtain the correct motion data, more than three markers should be attached to the paddle. To distinguish the front and back of the paddle, one additional marker needs to be attached to the front.

\subsection{Wireless Headsets}

The markers on the wireless headset are used for tracking the position of players. To increase the accuracy of captured motion data, there are four markers for each headset (Figure 5). We also propose creating a new VST file for the wireless headset.

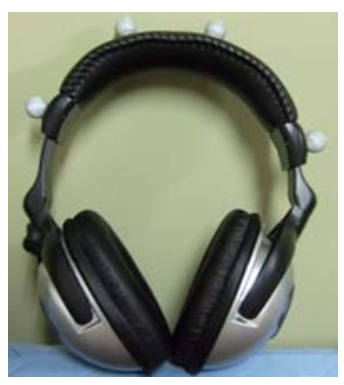

(a) The headset

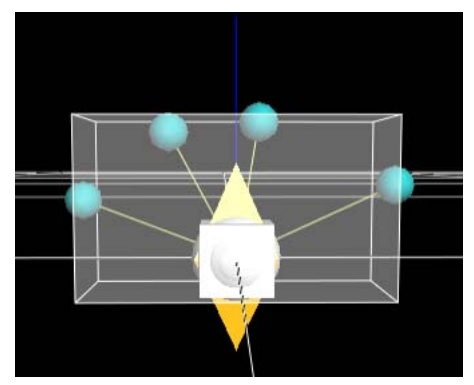

(b) VST of the headset
Figure 5. The wireless headset for multiplayer virtual ping-pong game

\section{Tactile Feedback Mechanism}

A dynamic game such as ping-pong can feel more realistic with the aid of feedback mechanisms. We use three feedback systems: visual, audio, and tactile. Visual and audio feedback (displaying the movement of a ball and paddles and making sounds when a ball collides with a paddle or table) is easier to implement than tactile feedback. Here we describe the tactile feedback system.

\subsection{Controller of the DC Motors}

For tactile feedback, we propose a new DC motor controller (Figure 6). This controller communicates to the game server via wireless Bluetooth and reacts to trigger the DC motors to vibrate depending on the position of collision virtual ball on the virtual paddle. The controller should be compact enough to be placed on the paddle. There are five DC motors which operate at 7.2 volts. These motors are controlled by a transistor and MCU (Micro Controller Unit), which both operate at 5.0 volts; voltage is reduced using a regulator. To minimize the size of the controller, we choose the ULN2803 array transistor and ATmega128 MCU. The usage of a push switch is to let the game server recognize that a player will serve a ball or choose game play options. The Bluetooth module on the controller communicates to the game server through a USB dongle. The Bluetooth module runs at 3.3 volts, thus a second regulator is needed.

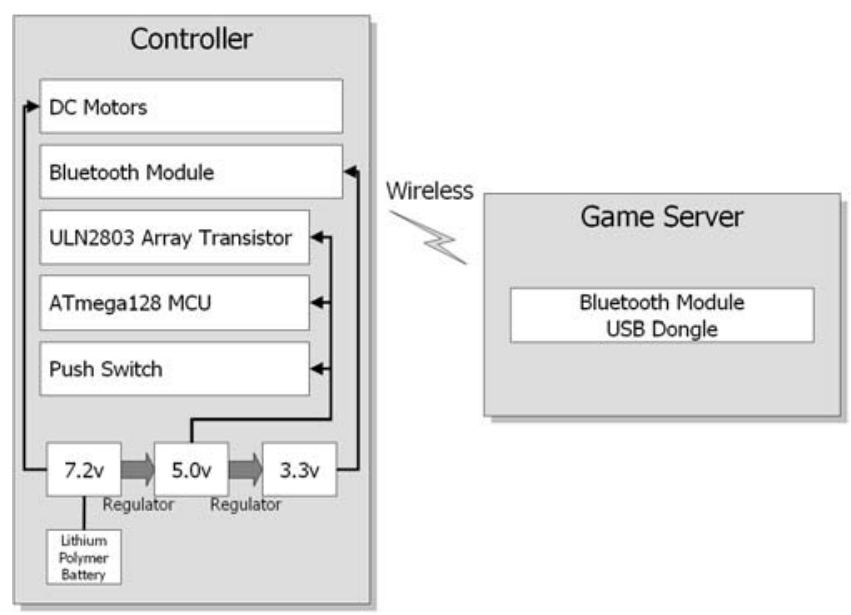

Figure 6. Design of the controller of the DC motors

Why we choose DC motors for tactile feedback is to avoid the latency. Step motors are very useful to control the level of vibration but spend more time in starting to vibrate than DC motors. Also the paddle is not too heavy because the weight of one DC motor is only about $20 \mathrm{~g}$.

\subsection{Tactile Feedback}

When the game server detects a collision between the paddle and ball, the server sends the message of the collision position to the controller of the DC motors in order to make one of the motors to vibrate. For collision detection, the binding box of the paddle is divided into four regions and the game server checks whether one of these four regions has collide against a ball or not using an ODE (Open Dynamics Engine) physics engine [3]. If the collision between a ball and one region is detected, the Bluetooth module on the controller receives a message for the corresponding region. This message is passed to the MCU that operates the appropriate motor. If the push switch on the controller is pressed, the MCU sends a signal to the Bluetooth module a message that a player will serve a ball (Figure 7). 


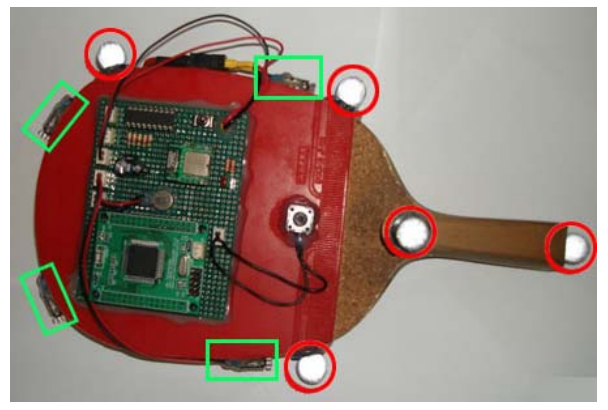

(a) Front of the paddle 1

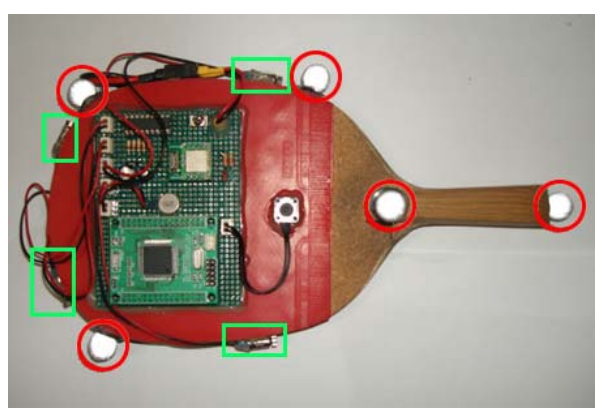

(d) Front of the paddle 2

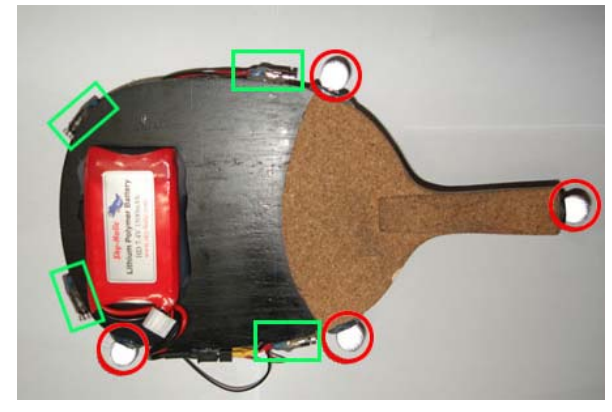

(b) Back of the paddle 1

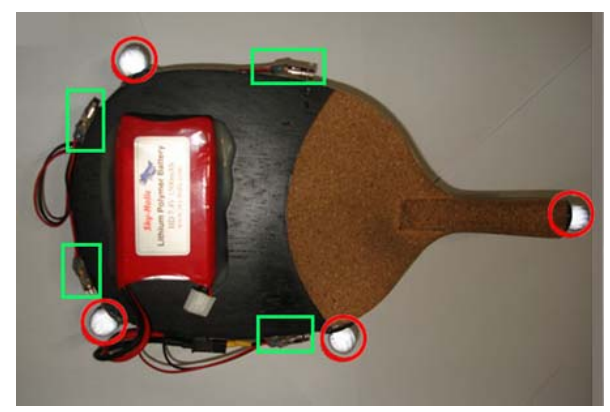

(e) Back of the paddle 2

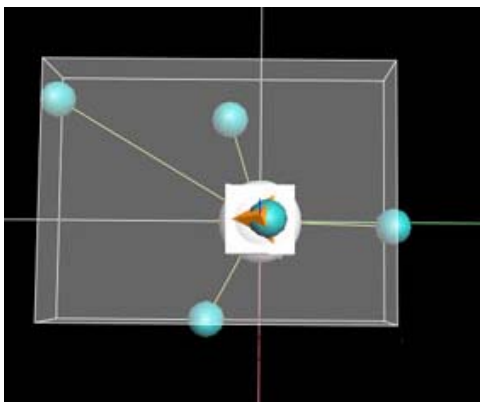

(c) VST of the paddle 1

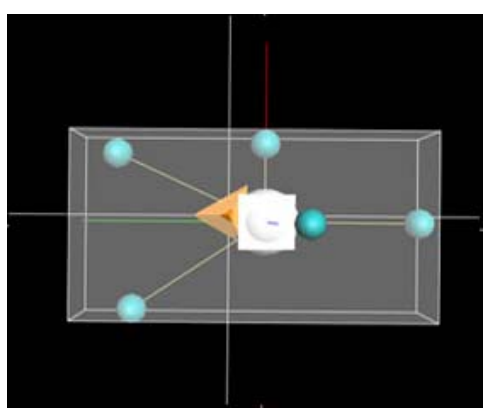

(f) VST of the paddle 2

Figure 4. The paddles for multi-player virtual ping-pong game: The first is for player 1 (the top row) and the second is for player 2 (the bottom row). The circles indicate optical markers and the squares indicate DC motors.

\section{Game Play in Practice}

Figure 8 shows the game interfaces: the left one is the opening page and the right one is the playing page. There are three views for a multi-player game: the top is the view of camera, the left is the view of the first player, and the right is the view of the second player. Figure 9 shows our multi-player virtual ping-pong game in play.

In the exhibition (Figure 1), instead of wireless headsets, speakers were used for audio feedback. The captured motion data using only the markers attached to the paddle were enough for people to enjoy our game. Test players evaluated our multi-player virtual ping-pong game as being very interesting. In contrast to other virtual ping-pong games, our tactile feedback system enhances player performance.

\section{Conclusion and Future Work}

In this paper we present a solution for a multi-player virtual ping-pong game. Optical markers are attached to the different positions of the paddles of each player. To capture the motion of these markers we built a new VST file. We also captured the motion of wireless headsets to help track the motion of players. For tactile feedback, we designed a new DC motor controller. The controller communicates to the game server through wireless Bluetooth. When the controller receives a message from the game server that the paddle has hit a ball, it triggers one of the DC motors to vibrate the paddle depending on the collision position.

In future work we want to bring our research to on-line games.

\section{Acknowledgement}

This study was supported by the grants to HEGA (Hallym Education for Game and Animation) NURI (New University for Regional Innovation). 


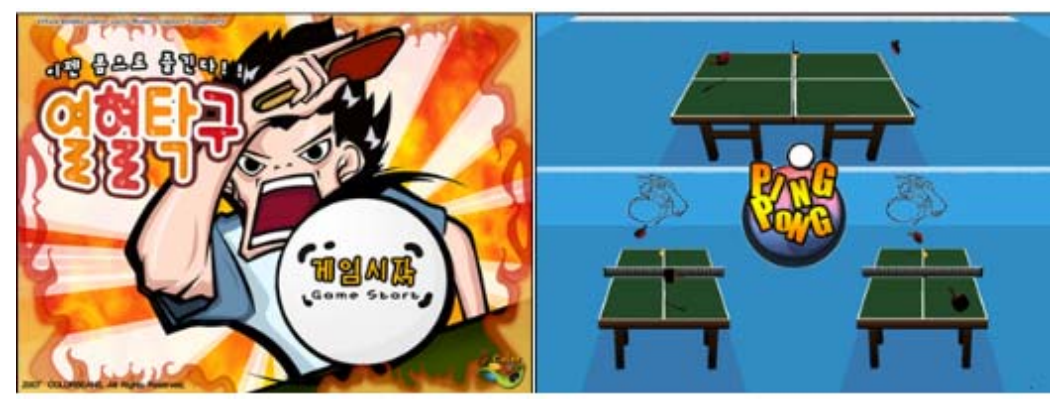

Figure 8. The interface of multi-player virtual ping-pong game

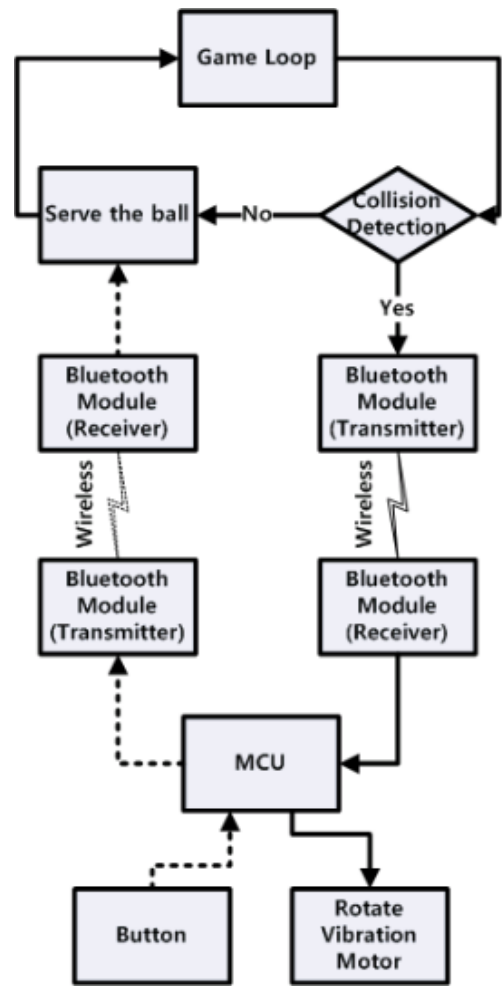

Figure 7. The flowchart for Bluetooth communication between the game server and the controller of the DC motors

\section{References}

[1] C. Bregler, C. Castiglia, J. DeVincenzo, L. Dubois, K. Feeley, T. Igoe, J. Meyer, M. Naimark, A. Postelnicu, M. Rabinovich, S. Rosenthal, K. Salen, J. Sudol, and B. Wright. Squidball: An Experiment in Large Scale Motion Capture and Game Design. In Proceedings of Intelligent Technologies for Interactive Entertainment (INTETAIN) 2005, pages 23-33, 2005.

[2] G. Brunnett, S. Rusdorf, and M. Lorenz. V-Pong: An Immer-

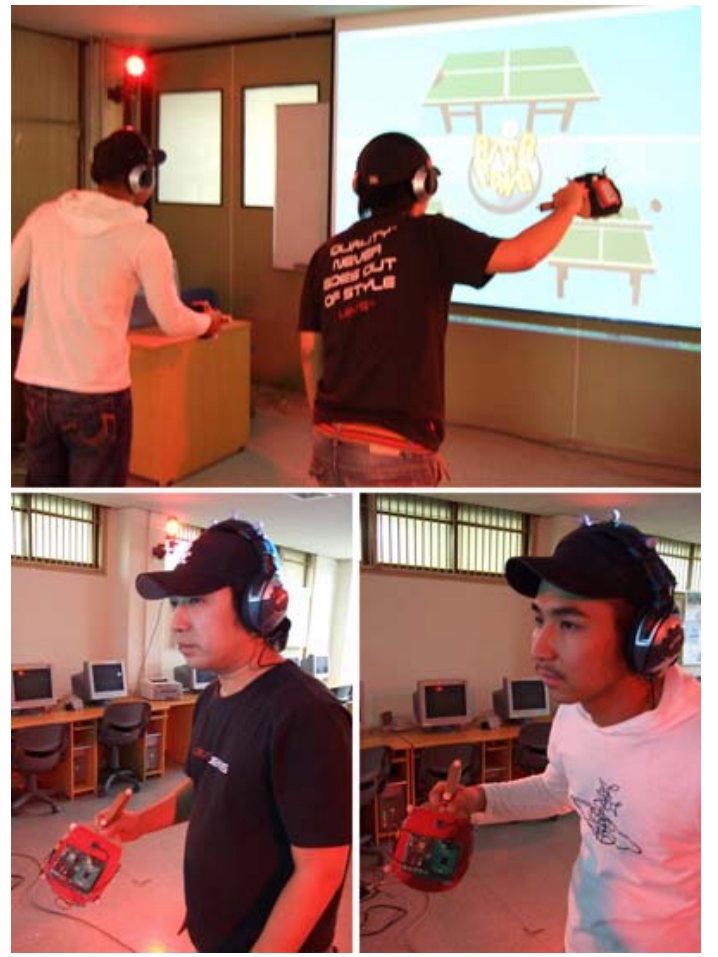

Figure 9. The screen-shot of multi-player virtual ping-pong game play

sive Table Tennis Simulation. IEEE Computer Graphics and Applications, 26(4):10-13, 2006.

[3] O. D. Engine. http://www.ode.org.

[4] T. Molet, A. Aubel, T. Capin, S. Carion, E. Lee, N. MagnenatThalmann, H. Noser, I. Pandzic, G. Sannier, and D. Thalmann. Anyone for Tennis? Presence, 8(2):140-156, 1999.

[5] VICON. http://www.vicon.com.

[6] J. Zagal, M. Nussbaum, and R. Rosas. A Model to Support the Design of Multiplayer Games. Presence, 9(5):448-462, 2000 . 